\title{
COVID-19 Pandemic and Reduced Physical Activity: Is There an Impact on Healthy and Asthmatic Children?
}

\author{
Giuliana Ferrante $^{1 *}$, Desiree Mollicone ${ }^{2 \dagger}$, Salvatore Cazzato ${ }^{3}$, Enrico Lombardi ${ }^{4}$, \\ Massimo Pifferi ${ }^{5}$, Attilio Turchetta ${ }^{6}$, Giancarlo Tancredi ${ }^{7}$ and Stefania La Grutta ${ }^{8}$ \\ on behalf of the Italian Pediatric Respiratory Society (IPRS)
}

\begin{abstract}
${ }^{1}$ Department of Health Promotion, Mother, and Child Care, Internal Medicine and Medical Specialties, University of Palermo, Palermo, Italy, ${ }^{2}$ Department of Anatomical and Histological Sciences, Legal Medicine and Orthopedics, Sapienza University of Rome, Rome, Italy, ${ }^{3}$ Department of Mother and Child Health, Salesi Children's Hospital, Ancona, Italy, ${ }^{4}$ Pediatric Pulmonary Unit, Meyer Pediatric University Hospital, Florence, Italy, ${ }^{5}$ Department of Paediatrics, University Hospital of Pisa, Pisa, Italy, ${ }^{6}$ Sport Medicine Unit, Bambino Gesù Children's Hospital, Rome, Italy, ${ }^{7}$ Pediatric Department, Sapienza University of Rome, Rome, Italy, ${ }^{8}$ National Research Council of Italy, Institute for Biomedical Research and Innovation (IRIB), Palermo, Italy
\end{abstract}

OPEN ACCESS

Edited by:

Giampaolo Ricci,

University of Bologna, Italy

Reviewed by:

Michael John Fayon,

Centre Hospitalier Universitaire de

Bordeaux, France

Michele Miraglia Del Giudice,

University of Campania Luigi

Vanvitelli, Italy

*Correspondence:

Giuliana Ferrante

giuliana.ferrante@unipa.it

†These authors have contributed equally to this work

Specialty section

This article was submitted to

Pediatric Pulmonology,

a section of the journal

Frontiers in Pediatrics

Received: 15 April 2021

Accepted: 20 August 2021

Published: 08 September 2021

Citation:

Ferrante G, Mollicone D, Cazzato $S$, Lombardi E, Pifferi M, Turchetta A, Tancredi G and La Grutta S (2021) COVID-19 Pandemic and Reduced Physical Activity: Is There an Impact on Healthy and Asthmatic Children?

Front. Pediatr. 9:695703.

doi: 10.3389/fped.2021.695703
Physical activity (PA) has been seen to improve asthma symptoms, lung function, and quality of life, as well as to reduce airway inflammation and bronchial responsiveness. As a consequence of the COVID-19 pandemic, the minimal amount of PA recommended by the World Health Organization -i.e., about $60 \mathrm{~min} /$ day of moderate-to-high intensity -is difficult to achieve for many children, particularly those living in urban areas. Short-term changes in PA because of the COVID-19 pandemic may become habitual, increasing the risk of adverse asthma outcomes in children. Indeed, prolonged home confinement during the COVID-19 pandemic reduces PA levels and increases sedentary behaviors, possibly impairing immune system function and increasing susceptibility to inflammatory diseases. However, there is limited evidence regarding the effects of lockdown due to COVID-19 on PA and sedentary behaviors in asthmatic children. Given that children stay longer indoors, indoor air pollution represents a major issue to consider during home confinement. This narrative review aims to summarize the available evidence about the impact of decreased PA and increased sedentary behaviors on children with asthma during the COVID-19 pandemic. In addition, strategies for supporting PA in children with asthma during the COVID-19 pandemic are suggested, also looking at the issue of indoor air quality.

Keywords: asthma, children, COVID-19, physical activity, sedentary behavior

\section{INTRODUCTION}

In December 2019, a public health emergency started due to an outbreak of novel coronavirus, now referred to as severe acute respiratory syndrome coronavirus 2 (SARS-CoV-2), in Wuhan, China (1). SARS-CoV-2 rapidly spread worldwide with high morbidity and mortality. On March 11, 2020, a global pandemic was declared by the World Health Organization (WHO). Government measures to counteract diffusion of the virus consist in hand and respiratory hygiene, physical distancing, remote working, home-quarantine and lock-down including closure of schools and gyms and limitation of recreational activities. During home-quarantine, ordinary daily routine has been radically modified with increasing risk of unhealthy habits (2). 
In particular, a general decline in physical activity (PA) has been reported. Indeed, social distancing and the obligation to stay at home potentially decrease opportunities for PA. The WHO 2020 guidelines on PA and sedentary behavior advised that children and adolescents should have as a minimum about $60 \mathrm{~min} /$ day of moderate-to-vigorous mostly aerobic PA, across the week. Moreover, they should limit the amount of sedentary time, particularly the amount of time watching TV and playing videogames (3). Nonetheless, due to the COVID-19 pandemic, the minimum recommended by WHO is difficult to achieve for many children, particularly those who live in urban areas (4).

Therefore, the "inactivity pandemic" emerged as one of the most relevant adverse effects due to the long period of homequarantine (5) and social isolation with potential detrimental consequences on physical well-being of children, particularly those with chronic diseases, disabilities and special health needs $(6,7)$.

Regular PA is recognized to be a good way to avoid diseases and keep healthy (8). In particular, there is evidence that training programs are beneficial in combating chronic respiratory diseases like asthma (9). It has been shown to improve asthma symptoms, lung function, and quality of life, and to reduce airway inflammation and bronchial responsiveness (1012). Moreover, PA is able to modulate the immune system function and can be protective against overweight and obesity, promoting a reduction of systemic inflammation (13).

Previous studies evaluated the effects of PA on childhood asthma in order to define its potential benefits. Systematic reviews have suggested that physical training (including running, swimming, and walking) may reduce airway inflammation in asthmatics (14) and that physical training (including running, gymnastics, cycling, swimming, weights and walking) has some positive effects on quality of life, without significantly improvement of resting lung function (15). A systematic review reported that exercise training (defined as training for $\geq 7$ days, $\geq 2$ times per week, $\geq 5$ training sessions in total) improved asthma symptoms, quality of life, exercise capacity, and lung function in adults and children (11). A more recent systematic review demonstrated that aerobic PA (any type of physical exercise training lasting at least $20 \mathrm{~min}$ per day, undertaken at least two times per week for a minimum duration of 4 weeks) improves asthma in children and adults by reducing the prevalence and frequency of nocturnal symptoms (16). In particular, among the studies performed on children, only one reported significant improvement in the nocturnal symptoms scores in both the groups experiencing two modalities of aerobic exercise (active video game and treadmill) for 8 weeks $(p=0.1)(17)$.

Worldwide, an alarming and increasing number of people are not sufficiently physically active; and it is feared that this number will increase in quarantine periods (18).

As decreased PA and increased sedentary behaviors during the quarantine period are expected to put a significant burden on children with chronic diseases, this narrative review aims to summarize the available evidence about the impact of decreased PA and increased sedentary behaviors on children with asthma during the COVID-19 pandemic. In addition, strategies for supporting PA in children with asthma during the COVID-19 pandemic also looking at the issue of indoor air quality.

\section{METHODS}

We examined original papers in English in the PubMed, Scopus and Embase databases using the following keywords, separately and in combination: asthma, COVID-19, physical activity, and children. The age range was birth to 18 years. There were no limitations regarding the date or country. We also delved into the WHO website and searched the reference lists of the retrieved articles.

\section{THE COVID-19 PANDEMIC: DECREASED PHYSICAL ACTIVITY AND INCREASED SEDENTARY BEHAVIORS IN NORMAL CHILDREN}

It is evident that during the COVID-19 pandemic, children and young people globally spent less time in PA. Reduced PA and increased sedentary behaviors are well-known risk factors for obesity and poor cardiorespiratory fitness among children, which can lead to serious health consequences. Moreover, PA is expected to have a beneficial impact on the immune system and the risk for upper respiratory tract infections (19). The current scenario of the COVID-19 pandemic undoubtedly represents an alarming situation for children, with effects on PA and sedentary behaviors, which were investigated by several studies (Table 1). Among studies conducted in Europe, one study in Italy examined the possibility that factors contributing to weight gain among children and adolescents with overweight and obesity were accentuated during lockdown. The authors found that time spent in sports activities decreased by $2.30 \pm 4.60 \mathrm{~h} /$ week $(p=0.003)$ whereas screen time increased by $4.85 \pm 2.40 \mathrm{~h} /$ day $(p<0.001)$. In addition, an inverse correlation was observed between changes in sport activities and changes in time spent in front of a screen, although at borderline significant level (20). A study conducted on Spanish pre-school children during the lockdown reported that PA decreased (mean difference, $\mathrm{MD}=-43.3 \mathrm{~min}$ per day) while sedentary time increased $(\mathrm{MD}=+50.2 \mathrm{~min}$ per day) (21). In addition to these data, an online survey administered in Spain to 516 parents serving to collect data about 860 preschoolers, children and adolescents showed a significant decrease in PA for all age subgroups before and during the confinement; in particular, the highest reduction of weekly minutes $[-120.4$ (SD 159.0)] was reported in children aged between 6 and 12 years. Also, screen time showed a significant increase for all participants and all age subgroups; the highest increase [+3.3 (SD 2.1) more daily hours] was observed for adolescents (13-16 years) (22). Conversely, Swedish pre-schoolers reported an increased in $\mathrm{PA}(\mathrm{MD}=+53 \mathrm{~min}$ per day) during the lockdown, but also spent more time watching TV or playing videogames $(\mathrm{MD}=$ +30 min per day). These findings could be linked to the fact that pre-schools, playgrounds, and parks in Sweden remained open and organized sports and activities for children continued even during the current pandemic; at the same time, the increased 
TABLE 1 | Effects of the COVID-19 pandemic on physical activity and sedentary behaviors in normal children.

\begin{tabular}{lllll}
\hline References Country Study type $\quad$ Study population Aim and study procedures $\quad$ Results & Limitations
\end{tabular}

\section{European studies}

Pietrobelli et al. Italy

(20)

a

Longitudinal

observational study

(baseline assessment:

May-June 2019;

second evaluation:

March-April 2020)

Alonso-Martínez Spain et al. (21)

López-Bueno Spain et al. (22)

Cross-sectional study (baseline assessment: 2019; second

evaluation: March-April 2020)

Cross-sectional study

conducted in

March-May 2020

Nyström et al. (23) Sweden

\section{International studies}

Sá et al. (24) Brazil

Bazil

May-June 2020) September-December
Cross-sectional study (baseline assessment: March-May 2019;

second evaluation:

Cross-sectional study conducted in

March-April 2020
41 children (mean To test the hypothesis that factors age 13.3.1 years) contributing to weight gain among children and adolescents with overweight and obesity are exacerbated during COVID-19 pandemic-associated lockdown by questionnaire

268 pre-schoolers To examine the effects of the aged 4-6 years COVID-19 lockdown on PA. sedentary time, and sleep assessed using accelerometry in the week in which the Spanish national state of alarm was declared $(n=21)$

860 children and To investigate the impact of the adolescents aged COVID-19 confinement on between 3 and 16 health-related behaviors in children years (mean age using an online survey administered $9.6 \pm 3.9$ years) to parents

82 children (mean To assess how movement behaviors age $4 \pm 0.5$ years) have been affected in pre-schoolers during the COVID-19 pandemic assessed using a questionnaire filled in by parents

806 children aged 0 to 12 years To valuate how families with children aged $<13$ years faced the period of social isolation resulting from the COVID-19 pandemic, especially regarding the time spent on $\mathrm{PA}$, games, outdoor activities and screen time by questionnaire

Dunton et al. (25) U.S.

Prospective study 211 children To examine the effects of the

Results

Limitations

Time spent in sports activities decreased by $2.30 \pm 4.60 \mathrm{~h} /$ week $(p=0.003)$; screen time increased by $4.85 \pm 2.40 \mathrm{~h} /$ day $(p<$ $0.001)$. An inverse correlation was observed between changes in sport activities and changes in time spent in front of a screen, although at borderline significant level $(r=-0.27$, borderline significant at $p=0.084$ )

Decrease in total PA [mean difference $(M D)=43.3$ min per day, 95\% Small sample size with $\mathrm{Cl}$ 68.1-18.5], and an increase in sedentary time ( $\mathrm{MD}=50.2 \mathrm{~min} \quad$ accelerometry data and a per day, $95 \% \mathrm{Cl} 17.1-83.3)$

Significant reduction of weekly minutes of PA during the confinement $(-102.5$, SD 159.6) $(p<0.001)$ and increase of daily hours of screen exposure $(2.9$, SD 2.1) $(p<0.001)$

PA, time spent outside on weekdays and weekend days, and screen Self-reported data by not time significantly increased $(+53 ;+124 ;+68 ;+30 \mathrm{~min} /$ day respectively, $p<0.001$ ).

Self-reported data, smal sample size short time of mor

Self-reported data, wide age range, convenience sampling validated questionnaire

Significant reduction in the percentage of total PA time (percentage of reported hours: $26.11 \%$ in children aged $0-2$ years; $19.56 \%$ in children aged $3-5$ years; $10.99 \%$ in children aged $6-9$ years; $9.77 \%$ in children aged 10-12 years) and an increase in total sedentary time (percentage of reported hours: $73.89 \%$ in children aged 0-2

years; $80.44 \%$ in children aged $3-5$ years; $89.01 \%$ in children aged $6-9$ years; $90.23 \%$ in children aged $10-12$ years) $(p<0.001)$

The most common physical activities during the early-COVID-19 Self-reported data, sample period were free play/unstructured activity (e.g., running around, tag) not representative

$(90 \%)$ and going for a walk (55\%). Children engaged in about $90 \mathrm{~min}$ compared to U.S. of school-related sitting and over $8 \mathrm{~h}$ of leisure-related sitting a day. demographic data and not Parents of older children (aged 9-13) vs. younger children (aged geographically equally $5-8)$ were half as likely $[\mathrm{OR}=0.54,95 \% \mathrm{Cl}(0.31,0.93)]$ to have one-unit change in the perception their children had done less sedentary behavior in past 7 days as compared to February 2020. The maximal oxygen uptake (VO2 max) in the post-COVID cohort was markedly lower than in the pre-COVID cohort (39.1 vs. 44.7 , $p=0.031$ ); the percentile of predicted VO2 max was significantly lower in the post-COVID cohort (95 vs. 105\%, $p=0.042$ ). There was a trend for the anaerobic threshold to be lower in the post-COVID cohort, even though it did not reach statistical significance (21.5 vs. $24.6, p=0.082$
Self-reported data distributed

Small sample size, comparisons of exercise performance not obtained on the same patient preand post-COVID but

through matched controls, different exercise protocols 
screen time observed could be connected to the fact that children stayed at home if they were symptomatic, allowing them to spend more time than usual in front of screens (23).

Similar findings were obtained by international studies conducted in Brazil and U.S. An online survey launched in Brazil to identify the behavior of children under 13 years during confinement due to the COVID-19 pandemic, indicated a significant reduction in the percentage of total PA time (percentage of reported hours: $26.11 \%$ in children aged $0-2$ years; $19.56 \%$ in children aged $3-5$ years; $10.99 \%$ in children aged 69 years; $9.77 \%$ in children aged $10-12$ years) and an increase in total sedentary time (percentage of reported hours: $73.89 \%$ in children aged $0-2$ years; $80.44 \%$ in children aged $3-5$ years; $89.01 \%$ in children aged $6-9$ years; $90.23 \%$ in children aged $10-12$ years) $(p<0.001)$ with age. $46.1 \%$ of parents reported that children were having much less PA, and 37\% that PA was less frequent than during the school period; $38 \%$ reported that screen time was higher than in regular school hours, and $36.9 \%$ that it was much higher (24). According to an online survey conducted in the U.S., about one child in three had online school lessons early on after the COVID-19 outbreak. Moreover, parents of children aged 9-13 years noticed more marked decreases in PA and larger increases in sedentary behaviors compared with parents of younger children (aged 5-8 years). Overall, children were reported to spend most of the time watching television/videos/movies, doing school-related work, and playing video games. Interestingly, whereas school-related sedentary time accounted for roughly 90 min per day, sitting for leisure activities accounted for over $8 \mathrm{~h}$ per day. In particular, older children and girls generally spent more time in a sedentary state than younger children and boys, which implies that these groups of children may be at greater risk of unhealthy lifestyle (25). In a singlecenter, retrospective case-control study, 10 healthy children who underwent cardiopulmonary exercise testing were compared to a matched cohort before the COVID-19-related lockdown began. The maximal oxygen uptake (VO2 max) in the post-COVID cohort was markedly lower than in the pre-COVID cohort (39.1 vs. $44.7, p=0.031$ ) and the percentile of predicted VO2 max was significantly lower in the post-COVID cohort ( 95 vs. $105 \%, p=$ 0.042 ), indicating a significant decline in the physical fitness of healthy children due to the COVID-19 pandemic and restrictions linked to it (26).

Overall, the available evidence suggests a decrease in PA generally associated with increased time spent in front of a screen by children and adolescents during the present pandemic. However, it should be pointed out that several limitations in methodology affected the published results; therefore, the available findings should be interpreted with caution.

\section{DECREASED PHYSICAL ACTIVITY AND INCREASED SEDENTARY BEHAVIORS IN ASTHMATIC CHILDREN, AND ITS IMPACT DURING THE COVID-19 PANDEMIC}

In the period of the COVID-19 pandemic, an objective decrease in PA levels has been observed in children with asthma. Using wearable sensors to continuously track personal location and PA, Kouis et al. assessed changes in mobility of asthmatic children in Cyprus and Greece, reporting an overall increase of time spent at home and a decrease of PA level (27). In Israel, a study using an electronic questionnaire submitted during lockdown (March-May 2020) to caregivers of children and adolescents with asthma and other chronic respiratory disorders demonstrated that patients aged $>5$ years had increased screen time, and decreased PA compared to their younger counterparts $(p=0.008$ and $p<0.001$, respectively) (28).

Studies investigating PA in childhood asthma showed that children with asthma tended to be less physically active than their healthy peers. Groth et al. found that adolescents with asthma in the U.S. had less PA than those without asthma. In particular, boys with asthma were less physically active than boys without asthma (high-intensity exercise: MD $-0.81, p<0.05$ ) and girls were less active than boys regardless of asthma status (highintensity exercise: $\mathrm{MD}-0.72, p<0.001)$. In addition, gender was found to be associated with sedentary behavior: screen time was higher in boys than girls (hours/day use pc or watch video games: MD: $-0.32, p<0.001)$ (29). Similarly, a study in Taiwan showed that children with moderate-to-severe asthma were less active than healthy children the same age. A correlation was found between PA and asthma severity level: children who had moderate or severe persistent asthma were more likely to be inactive (30).

These findings have not been confirmed by more recent studies, which showed similar levels of PA in children with asthma and healthy children. Within the UK Millennium Cohort, different levels of PA between children with asthma and controls were found but were not significant. However, it should be noted that in children with a recent asthma admission lower levels of total PA were observed than in controls. Overall, a low proportion (50-56\%) of children, whether healthy or asthmatic, were found to comply with the daily recommended PA guidelines (31). A very recent systematic review of 16 studies did not report differences in PA levels for young people with asthma compared to healthy peers; nonetheless, PA levels remained generally inadequate in most studies (32). Another systematic review of 28 studies suggested that children and adolescents with asthma have similar moderate to vigorous PA, steps/day, and sedentary time compared to controls (33).

PA has been found to ensure better asthma control in children (34). A study on Brazilian schoolchildren found that those with controlled asthma were more active than those whose asthma was uncontrolled $(p=0.032)$. Moreover, in more active schoolchildren asthma was more likely to be controlled $(\mathrm{OR}=1.5$; 95\%CI: 1.04-2.25) (35). Within the National Youth Fitness Survey, decreased aerobic fitness and increased sedentary time led to worse asthma outcomes including asthma attacks, wheeze with exercise, and wheeze with activity limitations, in children with doctor-diagnosed asthma (36). The effect of sedentary behaviors on childhood asthma outcomes needs to be further elucidated. Since sedentary children were found to present predominance of Th1 response in relation to active children, who instead showed higher levels of Interleukin 10 and higher regulatory $\mathrm{T}$ cell percentage, it could be hypothesized 
that regular PA may help to foster an anti-inflammatory profile. Consequently, prolonged social isolation during the COVID-19 pandemic, limiting PA, may lead to an increase in adiposity and susceptibility to inflammatory diseases (37).

Nonetheless, during the COVID-19 pandemic improved childhood asthma outcomes were observed, namely reduced acute attacks, emergency department visits, and hospitalizations as well as improved scores in asthma control measures and lung function. In particular, improved or unchanged asthma control during pandemic was reported by $90.2 \%$ of the participants to a multi-national cohort study including 1,054 children with asthma and 505 non-asthmatic children aged 4-18 years from 15 countries. These findings might be due to reduced exposure to outdoor asthma triggers, such as seasonal allergens and air pollutants, and increased treatment adherence (38). Noteworthy, social distancing and facemasks have been proposed to reduce respiratory viral infection, leading to less hospital admission for asthma exacerbation in adults. Wearing facemasks has been proved to be protective against respiratory infections which are a common trigger of asthma exacerbation (39). Indeed, a large study in Hong Kong reported that the number of admissions for asthma exacerbation was reduced by $53.2 \%$ (95\% CI $50.4-55.8 \%, p<0.0001$ ) in 2020 compared with past 5 years; admissions for asthma exacerbation decreased by $0.8 \%$ with every $1 \%$ increase in masking (95\% CI $0.8-0.9 \%, p<0.0001$ ) (40). However, Sykes et al. observed that, despite a decreased number of exacerbation-related admissions, adult patients with asthma showed a subjective decline in disease control and reported a negative impact on their mental health. Among those with severe asthma, 34.1\% reported their disease control to be worse and $18.3 \%$ reported the pandemic as having a negative effect on their mental health. The sedentary lifestyle during the COVID19 pandemic could be associated with worse disease outcomes in patients with severe asthma. However, the apparent discordance between perceived disease control and reduction in exacerbations requiring hospitalization in adults with severe asthma needs further investigation (41).

Among several factors that may have an impact on asthma severity and control, it should be acknowledged the discontinuation of in-person visits and the difficulty to perform lung function tests during the COVID-19 pandemic. A large online survey demonstrated that COVID-19 significantly impacted pediatric asthma services: $39 \%$ ceased in-person visits, $47 \%$ stopped accepting new patients, and 75\% limited patients' visits. Importantly, more than $90 \%$ of participating centers launched virtual online or telephone consultations, while $73 \%$ used a helpline to remotely assist their patients. Fortyeight percent of the participants considered virtual visits a suboptimal clinical encounter, whereas $42 \%$ of patients found them acceptable, or, even, as good as face-to-face visits (42). In the context of the current pandemic, we think that telemedicine may be helpful especially to patients with severe or uncontrolled asthma who might be at increased risk due to lack of monitoring caused by social distancing and lockdowns. Taquechel et al. demonstrated an increased use of telemedicine and decreased overall asthma encounters during the COVID-19 pandemic (43). Indeed, the current pandemic has accelerated the adoption of

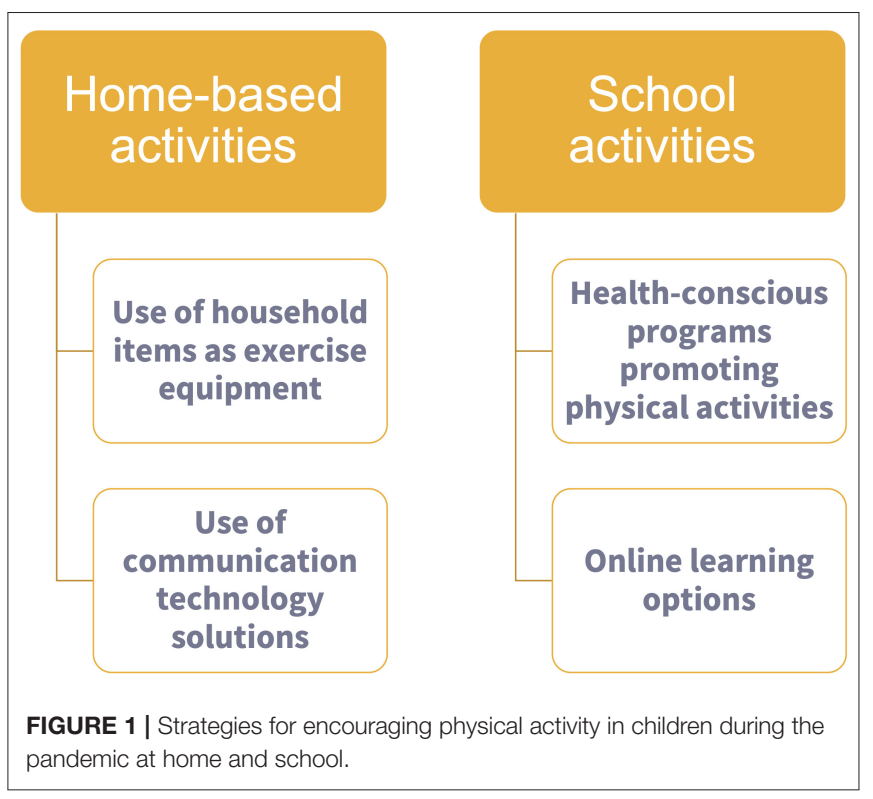

telemedicine in healthcare, which however represents a new and understudied care model. Overall, future research will be essential in order to implement technology-supported monitoring at home which should be targeted to the individual child and integrated to healthcare systems.

\section{STRATEGIES FOR SUPPORTING PHYSICAL ACTIVITY IN CHILDREN WITH ASTHMA DURING THE PANDEMIC}

Specific suggestions focused on decreasing sedentary behavior are vital, especially during a lockdown period, where it can be difficult to achieve moderate-vigorous PA (Figure 1). Promoting home-based activities that increase mobility can break sedentary behavior and increase levels of PA, thus enhancing health and well-being. Therefore, if the pandemic goes on, it will be fundamental to ensure that all children continue to have PA despite home confinement. Schools can integrate healthconscious programs by encouraging good personal hygiene, physical activities, appropriate diet, and good sleeping habits, in their curricula (44). For children having schooling online, it would be useful to provide opportunities for outdoor exercise without shareable equipment (45). However, measures that encourage and maintain regular PA in children cannot solely be dependent on school activities. Parents and caregivers should incorporate PA in children's daily home activities (including using electronic means to favor participation), but also the media and governments should provide regular messages promoting PA and healthy movement behaviors (46).

The recommendations provided so far for maintaining an active lifestyle during the pandemic mainly target the general population, and not so much vulnerable individuals like children with asthma $(47,48)$. Therefore, personalized PA programs with supervision are urgently required, possibly 


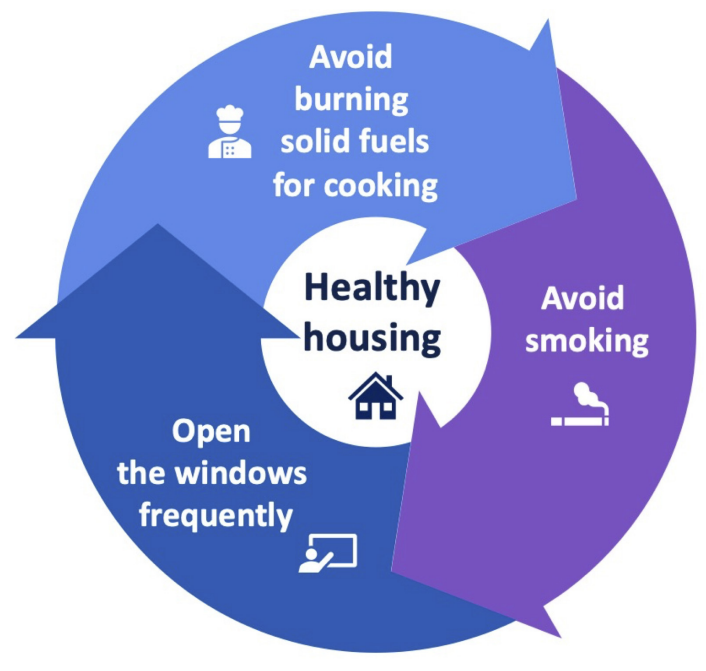

FIGURE 2 | Main suggestions for "healthy housing."

to be delivered and disseminated through communication technology solutions. Indeed, in the times of the pandemic, the use of digital tools to support and maintain PA could be particularly useful in children with chronic diseases (49). Recently, "serious games" (SGs), which are games not primarily oriented toward entertainment, enjoyment or fun, have provided new opportunities for constructive learning and training (50, 51). A very recent systematic review did not evidence that SGs improve PA levels in children with chronic diseases, likely due to small sample sizes and marked heterogeneity in study designs. Therefore, future research is required to evaluate the effectiveness of SGs in enhancing PA and healthy movement behaviors, especially in children with asthma (52). In a context of social distancing, Active video games (AVGs) can be an interesting alternative to increase PA levels in children (53). A controlled trial on children with moderate to severe asthma who were randomly allocated to either a video game group (VGG) or a treadmill group (TG) in an 8-week program with 2 weekly 40 min sessions found improvements in both groups with regard to asthma control and capacity. However, a marked reduction in exhaled nitric oxide levels was found in the VGG group [23.3 \pm 10.9 ppb post-VGG vs. $35.5 \pm 19.7$ ppb pre-VGG; $\Delta$ FeNO -13.2 (8.22) ppb, $p<0.05$ ], as well as a higher maximum energy expenditure $(7.31 \pm 1.64 \mathrm{cal} / \mathrm{min}$ in VGG vs. $5.68 \pm 1.27 \mathrm{cal} / \mathrm{min}$ in TG, $p<0.05$ ), suggesting that aerobic training linked to active videogames had a beneficial impact on asthma control, exercise capacity and airway inflammation (17). More recently, a crosssectional study on children submitted to a cardiopulmonary exercise test reported that active videogames can promote intense physical exercise and can be considered a motivating and efficient treatment modality for children with asthma (54).

In the pandemic period, children may experience negative feelings promoted by social isolation, which in turn may have an impact on their health. Indeed, a link between psychosocial factors and asthma outcomes including symptoms and lung function has been previously demonstrated in young people (55). Therefore, it could be speculated that the use of AVGs implementing PA and exercise with digital games may positively impact psychosocial variables and health (56). Obviously, recommendations for promoting healthy movement behaviors during the COVID-19 pandemic should consider equity and minimal equipment, especially for children living in communities with little access to the internet (46).

\section{PERFORMING PHYSICAL ACTIVITY AT HOME DURING THE COVID-19 PANDEMIC: THE ISSUE OF INDOOR AIR QUALITY}

As a consequence of governmental restrictions across the world due to the pandemic, time spent indoors is growing; therefore, exposure to certain indoor pollutants, such as tobacco smoke and other combustion products, has dramatically increased (57, 58). Indeed, homes often afford little space in relation to the number of inhabitants and often do not have adequate systems for ventilation and air renewal (59). In addition, human activities such as cooking (especially frying) and the movement of people in the room are significant contributors to concentrations of indoor pollutants (60). In particular, during aerobic exercise, inhaled air predominantly enters through the mouth, and respiratory uptake of airborne contaminants increases, with greater penetration to the lower lung regions. Furthermore, there is an association between the number of exercising individuals in confined environments and concentrations of coarse resuspended aerosols $\left(\mathrm{PM}_{10-2.5}\right)$ (61). In addition, during the COVID-19 pandemic the increasing use of household cleaning products and disinfectants to reduce the potential of viral infection represented a particularly significant source of indoor pollution. As a consequence, a study in Spain reported that mean daily Volatile Organic Compound concentration rose by $37-559 \%$, although this finding needs further research to quantify its actual impact on health (59). The increased intensity of time spent at home generates rising pollutant levels which are not always counteracted by ventilation in the home environment, due to its reduced effectiveness and/or to voluntary limitations. Overall, these factors may produce adverse effects on respiratory health, especially in vulnerable individuals. Indeed, increasing evidence has shown that the exposure of children to environmental indoor stressors is associated with respiratory conditions including wheezing, asthma, and rhinitis (62). It should also be pointed out that co-exposure to indoor allergens may aggravate airway inflammation, resulting in worsening of symptoms in sensitized children (63). The multifactorial routes of exposure to indoor pollutants should therefore be considered, also with a view to future confinement episodes for possible new waves of SARS-CoV-2.

Some suggestions to improve indoor air quality may include the following: cooking in a healthier way in order to emit less pollutants; avoiding burning solid fuels; opening the windows frequently and using a kitchen ventilator when cooking, if possible; refraining from smoking at home (64); enhancing awareness about indoor air quality; and implementing 
educational programs at the community level (Figure 2). Increasing awareness about "healthy housing" may translate into preventive action by incorporating best practices to reduce the burden of indoor exposures on health, especially in vulnerable populations. Rapid developments in the scientific field of housing technology will likely help to achieve better indoor air quality in the near future to ensure safer indoor living conditions (65).

\section{CONCLUSIONS}

The available evidence suggests a decrease in PA generally associated with increased time spent in front of a screen in both healthy children and adolescents during the pandemic. Decreased PA levels and increased sedentary behaviors have also been observed in children with asthma. Promoting home-based activities that increase mobility can lessen sedentary behavior and increase levels of PA, thus improving asthma outcomes in children. Therefore, if the pandemic goes on, it will be

\section{REFERENCES}

1. Wang C, Horby PW, Hayden FG, Gao GF. A novel coronavirus outbreak of global health concern. Lancet. (2020) 395:4703. doi: $10.1016 /$ S0140-6736(20)30185-9

2. Poletti M, Raballo A. Evidence on school closure and children's social contact: useful for coronavirus disease (COVID-19)? Euro Surveill. (2020) 25:2000758. doi: 10.2807/1560-7917.ES.2020.25.17.2000758

3. Bull FC, Al-Ansari SS, Biddle S, Borodulin K, Buman MP, Cardon G, et al. World Health Organization 2020 guidelines on physical activity and sedentary behaviour. Br J Sports Med. (2020) 54:1451-62. doi: 10.1136/bjsports-2020-102955

4. Ashikalli L, Carroll W, Johnson C. The indirect impact of COVID-19 on child health. Paediatr Child Health. (2020) 30:430-7. doi: 10.1016/j.paed.2020.09.004

5. Hall G, Laddu DR, Phillips SA, Lavie CJ, Arena R. A tale of two pandemics: How will COVID-19 and global trends in physical inactivity and sedentary behavior affect one another? Progr Cardiovasc Dis. (2021) 64:108-10. doi: 10.1016/j.pcad.2020.04.005

6. Hemphill NM, Kuan MT, Harris KC. Reduced physical activity during COVID-19 pandemic in children with congenital heart disease. Can J Cardiol. (2020) 36:1130-4. doi: 10.1016/j.cjca.2020.04.038

7. Armitage R, Nellums LB. The COVID-19 response must be disability inclusive. Lancet Public Health. (2020) 5:e257. doi: 10.1016/S2468-2667(20)30076-1

8. Latimer-Cheung AE, Toll BA, Salovey P. Promoting increased physical activity and reduced inactivity. Lancet. (2013) 381:114. doi: 10.1016/S0140-6736(13)60045-8

9. Moreira A, Bonini M, Pawankar R, Anderson SD, Carlsen K-H, Randolph C, et al. A World Allergy Organization international survey on physical activity as a treatment option for asthma and allergies. World Allergy Organ J. (2014) 7:34. doi: 10.1186/1939-4551-7-34

10. Moreira A, Delgado L, Haahtela T, Fonseca J, Moreira P, Lopes C, et al. Physical training does not increase allergic inflammation in asthmatic children. Eur Respir J. (2008) 32:1570-5. doi: 10.1183/09031936.00171707

11. Eichenberger PA, Diener SN, Kofmehl R, Spengler CM. Effects of exercise training on airway hyperreactivity in asthma: a systematic review and metaanalysis. Sports Med. (2013) 43:1157-70. doi: 10.1007/s40279-013-0077-2

12. Del Giacco SR, Garcia-Larsen V. Aerobic exercise training reduces bronchial hyper-responsiveness and serum pro-inflammatory vital to ensure that children with asthma continue engaging in PA despite home confinement. Parents and caregivers should make PA a part of children's daily routine at home, but the media and governments should also provide regular messages to promote PA and healthy movement behaviors. Personalized PA programs with supervision are required, possibly to be delivered through communication technology solutions. Indeed, in these pandemic times, the use of digital tools to support and maintain PA could be particularly useful in children with chronic diseases. Improving indoor air quality is crucial to ensure a safe environment where children with asthma can perform PA.

\section{AUTHOR CONTRIBUTIONS}

DM, GF, GT, and SL: conceptualization, methodology, and writing original draft. GF and SL: review and editing. AT, GT, EL, MP, and SC: supervision. All authors have read and agreed to the published version of the manuscript.

cytokines in patients with asthma. BMJ Evidence-Based Med. (2016) 21:70-70. doi: 10.1136/ebmed-2015-110260

13. Izadpanah A, Barnard RJ, Almeda AJE, Baldwin GC, Bridges SA, Shellman ER, et al. A short-term diet and exercise intervention ameliorates inflammation and markers of metabolic health in overweight/obese children. Am J Physiol Endocrinol Metab. (2012) 303:E542-50. doi: 10.1152/ajpendo.001 90.2012

14. Pakhale S, Luks V, Burkett A, Turner L. Effect of physical training on airway inflammation in bronchial asthma: a systematic review. BMC Pulm Med. (2013) 13:38. doi: 10.1186/1471-2466-13-38

15. Carson KV, Chandratilleke MG, Picot J, Brinn MP, Esterman AJ, Smith BJ. Physical training for asthma. Cochrane Database Syst Rev. (2013) 9:CD001116. doi: 10.1002/14651858.CD001116.pub4

16. Francisco CO, Bhatawadekar SA, Babineau J, Reid WD, Yadollahi A. Effects of physical exercise training on nocturnal symptoms in asthma: Systematic review. PLoS ONE. (2018) 13:e0204953. doi: 10.1371/journal.pone.0204953

17. Gomes ELFD, Carvalho CRF, Peixoto-Souza FS, Teixeira-Carvalho EF, Mendonca JFB, Stirbulov R, et al. Active video game exercise training improves the clinical control of asthma in children: randomized controlled trial. PLoS ONE. (2015) 10:0135433. doi: 10.1371/journal.pone.0135433

18. Sato $\mathrm{H}$, Inoue $\mathrm{S}$, Fukushima $\mathrm{N}$, Kikuchi $\mathrm{H}$, Takamiya T, Tudor-Locke $\mathrm{C}$, et al. Lower youth steps/day values observed at both high and low population density areas: a cross-sectional study in metropolitan Tokyo. BMC Public Health. (2018) 18:1-14. doi: 10.1186/s12889-018-6028-y

19. Timmons BW. Exercise and immune function in children. Am J Lifestyle Med. (2007) 1:59-66. doi: 10.1177/1559827606294851

20. Pietrobelli A, Pecoraro L, Ferruzzi A, Heo M, Faith M, Zoller T, et al. Effects of COVID-19 lockdown on lifestyle behaviors in children with obesity living in Verona, Italy: a longitudinal study. Obesity. (2020) 28:13825. doi: 10.1002/oby.22861

21. Alonso-Martínez AM, Ramírez-Vélez R, García-Alonso Y, Izquierdo M, García-Hermoso A. Physical activity, sedentary behavior, sleep and selfregulation in spanish preschoolers during the COVID-19 lockdown. Int $J$ Environ Res Public Health. (2021) 18:693. doi: 10.3390/ijerph18020693

22. López-Bueno R, López-Sánchez GF, Casajús JA, Calatayud J, Gil-Salmerón A, Grabovac I, et al. Health-related behaviors among school-aged children and adolescents during the Spanish Covid-19 confinement. Front Pediatr. (2020) 8:573. doi: $10.3389 /$ fped.2020.00573

23. Nyström CD, Alexandrou C, Henström M, Nilsson E, Okely AD, El Masri SW, et al. International study of movement behaviors in the early years (SUNRISE): 
results from SUNRISE Sweden's pilot and COVID-19 study. Int J Environ Res Public Health. (2020) 17:8491. doi: 10.3390/ijerph17228491

24. Sá CDSC, Pombo A, Luz C, Rodrigues LP, Cordovil R. Covid19 social isolation in brazil: effects on the physical activity routine of families with children. Rev Paul Pediatr. (2021) 39:e2020159. doi: 10.1590/1984-0462/2021/39/2020159

25. Dunton GF, Do B, Wang SD. Early effects of the COVID-19 pandemic on physical activity and sedentary behavior in children living in the US. $B M C$ Public Health. (2020) 20:1-13. doi: 10.1186/s12889-020-09429-3

26. Dayton JD, Ford K, Carroll SJ, Flynn PA, Kourtidou S, Holzer RJ. The Deconditioning Effect of the COVID-19 Pandemic on Unaffected Healthy Children. Pediatric Cardiol. (2021) 42:554-9. doi: 10.1007/s00246-020-02513-w

27. Panayiotis K, Antonis M, Pinelopi A, Emmanouil G, Eleni M, Dimitriou H, et al. Use of wearable sensors to assess compliance of asthmatic children in response to lockdown measures for the COVID-19 epidemic. Sci Rep. (2021) 11:5895. doi: 10.1038/s41598-021-85358-4

28. Cahal M, Amirav I, Diamant N, Be'er M, Besor O, Lavie M. Real-time effects of COVID-19 pandemic lockdown on pediatric respiratory patients. Pediatr Pulmonol. (2021) 56:1401-8. doi: 10.1002/ppul.25310

29. Groth SW, Rhee H, Kitzman H. Relationships among obesity, physical activity and sedentary behavior in young adolescents with and without lifetime asthma. J Asthma. (2016) 53:19-24. doi: 10.3109/02770903.2015.1063646

30. Lam K-M, Yang Y-H, Wang L-C, Chen S-Y, Gau B-S, Chiang B-L. Physical activity in school-aged children with asthma in an urban city of Taiwan. Pediatr Neonatol. (2016) 57:333-7. doi: 10.1016/j.pedneo.2015.05.003

31. Pike KC, Griffiths LJ, Dezateux C, Pearce A. Physical activity among children with asthma: cross-sectional analysis in the UK millennium cohort. Pediatr Pulmonol. (2019) 54:962-9. doi: 10.1002/ppul.24314

32. Mackintosh KA, McNarry MA, Berntsen S, Steele J, Sejersted E, Westergren T. Physical activity and sedentary time in children and adolescents with asthma: a systematic review and meta-analysis. Scand J Med Sci Sports. (2021) 31:1183-95. doi: 10.1111/sms.13937

33. Vasconcello-Castillo L, Torres-Castro R, Sepúlveda-Cáceres N, AcostaDighero R, Miranda-Aguilera S, Puppo H, et al. Levels of physical activity in children and adolescents with asthma: a systematic review and meta-analysis. Pediatr Pulmonol. (2021) 56:1307-23. doi: 10.1002/ppul.25293

34. Cordova-Rivera L, Gibson PG, Gardiner PA, McDonald VM, A. systematic review of associations of physical activity and sedentary time with asthma outcomes. J Allergy Clin Immunol Pract. (2018) 6:1968-81. doi: 10.1016/j.jaip.2018.02.027

35. dos Santos AP, Strassburger MJ, Roncada C, Stein RT, Pitrez PM, Strassburger SZ. Effect of physical activity on asthma control in schoolchildren. Einstein (São Paulo). (2020) 18:eAO4936. doi: 10.31744/einstein_journal/2020AO4936

36. Lu KD, Forno E, Radom-Aizik S, Cooper DM. Low fitness and increased sedentary time are associated with worse asthma-The National Youth Fitness Survey. Pediatr Pulmonol. (2020) 55:1116-23. doi: 10.1002/ppul.24678

37. Merlin M, de Oliveira HH, Passos MEP, Momesso CM, de Oliveira LC dos S, Santana JE, et al. Relationship between children physical activity, inflammatory mediators and lymphocyte activation: possible impact of social isolation (COVID-19). Sport Sci Health. (2020). doi: 10.1007/s11332-020-00719-2. [Epub ahead of print].

38. Papadopoulos NG, Mathioudakis AG, Custovic A, Deschildre A, Phipatanakul W, Wong G, et al. Childhood asthma outcomes during the COVID-19 pandemic: findings from the PeARL multinational cohort. Allergy. (2021) 76:1765-75. doi: 10.1101/2020.10.27.20219436

39. Liuzzo Scorpo M, Ferrante G, La Grutta S. An overview of asthma and COVID-19: protective factors against SARS-COV-2 in pediatric patients. Front Pediatr. (2021) 9:661206. doi: 10.3389/fped.2021.661206

40. Chan KPF, Kwok WC, Ma TF, Hui CH, Tam TC, Wang JK, et al. Territory-wide study on hospital admissions for asthma exacerbation in COVID-19 pandemic. Ann Am Thorac Soc. (2021). doi: 10.1513/AnnalsATS.202010-1247OC. [Epub ahead of print].

41. Sykes DL, Faruqi S, Holdsworth L, Crooks MG. Impact of COVID-19 on COPD and asthma admissions, and the pandemic from a patient's perspective. ERJ Open Res. (2021) 7:00822-2020. doi: 10.1183/23120541.00822-2020

42. Papadopoulos NG, Custovic A, Deschildre A, Mathioudakis AG, Phipatanakul W, Wong G, et al. Impact of COVID-19 on pediatric asthma: practice adjustments and disease burden. J Allergy Clin Immunol Pract. (2020) 8:2592-9.e3. doi: 10.1016/j.jaip.2020.06.001

43. Taquechel K, Diwadkar AR, Sayed S, Dudley JW, Grundmeier RW, Kenyon CC, et al. Pediatric asthma health care utilization, viral testing, and air pollution changes during the COVID-19 pandemic. J Allergy Clin Immunol Pract. (2020) 8:3378-87.e11. doi: 10.1016/j.jaip.2020.07.057

44. Brazendale K, Beets MW, Weaver RG, Pate RR, Turner-McGrievy GM, Kaczynski AT, et al. Understanding differences between summer vs. school obesogenic behaviors of children: the structured days hypothesis. Int J Behav Nutr Phys Act. (2017) 14:1-14. doi: 10.1186/s12966-017-0555-2

45. Pavlovic A, DeFina L, Natale B, Thiele S, Walker T, Craig D, et al. Keeping children healthy during and after COVID-19 pandemic: meeting youth physical activity needs. BMC Public Health. (2021) 21:485. doi: $10.1186 / \mathrm{s} 12889-021-10545-\mathrm{x}$

46. Guan H, Okely AD, Aguilar-Farias N. del Pozo Cruz B, Draper CE, El Hamdouchi A, et al. Promoting healthy movement behaviours among children during the COVID-19 pandemic. Lancet Child Adolesc Health. (2020) 4:416-8. doi: 10.1016/S2352-4642(20)30131-0

47. Margaritis I, Houdart S, El Ouadrhiri Y, Bigard X, Vuillemin A, Duché P. How to deal with COVID-19 epidemic-related lockdown physical inactivity and sedentary increase in youth? Adaptation of Anses' benchmarks. Arch Public Health. (2020) 78:1-6. doi: 10.1186/s13690-020-00432-z

48. Bentlage E, Ammar A, How D, Ahmed M, Trabelsi K, Chtourou H, et al. Practical recommendations for maintaining active lifestyle during the COVID-19 pandemic: a systematic literature review. Int J Environ Res Public Health. (2020) 17:6265. doi: 10.3390/ijerph17176265

49. Ferrante G, Licari A, Marseglia GL, La Grutta S. Digital health interventions in children with asthma. Clin Exp Allergy. (2021) 51:212-20. doi: 10.1111/cea.13793

50. Licari A, Ferrante G, Marseglia GL, Corsello G, La Grutta S. What is the impact of innovative electronic health interventions in improving treatment adherence in asthma? The pediatric perspective. J Allergy Clin Immunol Pract. (2019) 7:2574-9. doi: 10.1016/j.jaip.2019.08.008

51. Michael DR, Chen SL. Serious Games: Games that Educate, Train, and Inform. Boston, MA: Muska and Lipman/Premier-Trade (2005).

52. Bossen D, Broekema A, Visser B, Brons A, Timmerman A, Van EttenJamaludin F, et al. Effectiveness of serious games to increase physical activity in children with a chronic disease: systematic review with meta-analysis. J Med Internet Res. (2020) 22:e14549. doi: 10.2196/14549

53. González CS, Gómez N, Navarro V, Cairós M, Quirce C, Toledo P, et al. Learning healthy lifestyles through active videogames, motor games and the gamification of educational activities. Comput Hum Behav. (2016) 55:52951. doi: 10.1016/j.chb.2015.08.052

54. Barreto-Mendonça JF, de Freitas Dantas EL. The intensity of physical activity in asthmatic children during active video game playing. EMJ Allergy Immunol. (2019) 4:101-7.

55. Resztak JA, Farrell AK, Mair-Meijers HE, Alazizi A. Psychosocial experiences modulate asthma-associated genes through gene. Annu Rev Public Health. (2005) 26:469-500.

56. Brilliant TD, Nouchi R, Kawashima R. Does video gaming have impacts on the brain: evidence from a systematic review. Brain Sci. (2019) 9:251. doi: 10.3390/brainsci9100251

57. Vardoulakis S, Giagloglou E, Steinle S, Davis A, Sleeuwenhoek A, Galea KS, et al. Indoor exposure to selected air pollutants in the home environment: A systematic review. Int J Environ Res Public Health. (2020) 17:124. doi: 10.3390/ijerph17238972

58. Van Tran V, Park D, Lee Y. Indoor air pollution, related human diseases, and recent trends in the control and improvement of indoor air quality. Int J Environ Res Public Health. (2020) 17:2927. doi: 10.3390/ijerph170 82927

59. Domínguez-Amarillo S, Fernández-Agüera J, Cesteros-García S, GonzálezLezcano RA. Bad air can also kill: Residential indoor air quality and pollutant exposure risk during the covid-19 crisis. Int J Environ Res Public Health. (2020) 17:1-34. doi: 10.3390/ijerph17197183

60. Jones NC, Thornton CA, Mark D, Harrison RM. Indoor/outdoor relationships of particulate matter in domestic homes with roadside, urban and rural locations. Atmos Environ. (2000) 34:2603-12. doi: 10.1016/S1352-2310(99)00489-6 
61. Braniš M, Šafránek J, Hytychová A. Indoor and outdoor sources of size-resolved mass concentration of particulate matter in a school gymimplications for exposure of exercising children. Environ Sci Pollut Res Int. (2011) 18:598-609. doi: 10.1007/s11356-010-0405-0

62. Tham KW. Indoor air quality and its effects on humans-A review of challenges and developments in the last 30 years. Energy Build. (2016) 130:637-50. doi: 10.1016/j.enbuild.2016.08.071

63. Yucel E, Suleyman A, Hizli Demirkale Z, Guler N, Ulker Tamay Z, Ozdemir C. 'Stay at home': Is it good or not for house dust mite sensitized children with respiratory allergies? Pediatr Allergy Immunol. (2021) 32:96370. doi: 10.1111 pai.13477

64. Du W, Wang G. Indoor air pollution was nonnegligible during covid-19 lockdown. Aerosol Air Qual Res. (2020) 20:18515. doi: 10.4209/aaqr.2020.06.0281

65. Schieweck A, Uhde E, Salthammer T, Salthammer LC, Morawska L, Mazaheri M, et al. Smart homes and the control of indoor air quality. Renew Sustain Energy Rev. (2018) 94:705-18. doi: 10.1016/j.rser.2018. 05.057
Conflict of Interest: The authors declare that the research was conducted in the absence of any commercial or financial relationships that could be construed as a potential conflict of interest.

Publisher's Note: All claims expressed in this article are solely those of the authors and do not necessarily represent those of their affiliated organizations, or those of the publisher, the editors and the reviewers. Any product that may be evaluated in this article, or claim that may be made by its manufacturer, is not guaranteed or endorsed by the publisher.

Copyright (®) 2021 Ferrante, Mollicone, Cazzato, Lombardi, Pifferi, Turchetta, Tancredi and La Grutta. This is an open-access article distributed under the terms of the Creative Commons Attribution License (CC BY). The use, distribution or reproduction in other forums is permitted, provided the original author(s) and the copyright owner(s) are credited and that the original publication in this journal is cited, in accordance with accepted academic practice. No use, distribution or reproduction is permitted which does not comply with these terms. 